\title{
Flora XeROfítica DE LA Cuenca DE APURÍMAC: Limatambo - Gurahuasi
}

\author{
Alfonso Aréstegui Pezúa, Alfredo Tupayachi Herrera, Lourdes Acurio Mendoza, \\ José E. Yabarrena Urday, Violeta Zamalloa Acurio y Jim Farfán Vargas
}

\section{INTRODUCGIÓN}

El sur peruano, al igual que todo el territorio, presenta una topografia muy accidentada debido fundamentalmente a la presencia de la Cordillera de los Andes que atraviesa el país de Sur a Norte, siguiendo una dirección paralela al litoral Pacífico. La cordillera divide al país en tres macro-regiones: una zona desértica, ubicada en el lado occidental disectada por ríos costeros; la región andina, formada por numerosos valles, quebradas, cañones, punas y jalca etc., y la zona oriental, caracterizada por bosques húmedos que descienden a la Amazonía. Los bosques secos tradicionalmente semideciduos son de amplia distribución en los neo trópicos; bosques secos de extensión considerable que se encuentran enclavados en ciertas localidades de los valles interandinos del Sur Este del Perú, como los de Apurímac, Pachachaca, Pampas y Urubamba; la sabana pluvifolia de La Convención, Paucartambo o Mapacho (Weberbauer, 1945; Vargas C., 1972; Galiano S., 1990; Núñez V. et al, 2002). Sin embargo, dentro de la Región Sur Andina existen zonas desérticas caracterizadas por ausencia del recurso hídrico y suelos agrícolas, con gran diversidad de flora adaptadas a condiciones xéricas que soportan grandes sequías.

En una visión retrospectiva, el paisaje de Limatambo -Cúnyaq- Curahuasi, comparada con la realidad concreta de nuestros días, permite constatar cambios marcados por causas antropogénicas y climáticas. Las actividades antrópicas acarrean profundos impactos en el ambiente natural; así por ejemplo, los cambios de uso de la tierra donde se desarrollaban los bosques secos, ahora con la ampliación de la frontera agrícola para cultivos de frutales, cereales, hortalizas y cultivos industriales como la caña de azúcar, han causado un riesgo en el balance ecológico de la zona; a esto se suman las obras civiles (canales de irrigación, carreteras y puentes), la tala ilegal de los bosques desde décadas pasadas para la obtención de leña y carbón que han impactado negativamente a estos frágiles ecosistemas, exponiéndolos a los suelos sin cubierta vegetal a la acción eólica y pluvial erosivas en áreas de pendientes con bosques secos muy ralos, acelerando de esta forma el proceso de desertización.

La presente investigación se realizó durante los años 2007 y 2008 en las dos estaciones climáticas, evaluando la flora xerofítica de la cuenca del Apurímac, en el trecho comprendido entre Limatambo (2700 m) - Cúnyaq (1950 m) y Curahuasi $(2750 \mathrm{~m})$, a través de una gradiente altitudinal por el método de transecto en banda a ambos lados de río Blanco y Apurímac, que nos permitió evaluar la diversidad de flora arbórea, arbustiva, herbácea, cactácea y suculenta que permita determinar los valores de los índices poblacionales y establecer las especies endémicas de la zona.

En la zona de estudio se ha encontrado la siguiente variabilidad: 42 familias botánicas, 100 géneros y 122 especies, con dominancia de Asteraceae y Poaceae. De acuerdo con los resultados las especies: Opuntia ficus indica (L.) Millar, Furcraea andina Treleace, Guilleminea densa (Willdenow) Moquin, Paspahum ceresioides Carrillo, y Vargaseniopsis vargasii Lyman B. Smith, que presentan mayores índices de valor de importancia para las 5 parcelas establecidas en ambas márgenes del río Apurímac. 


\section{Problema objeto de investigación}

En los últimos años los ecosistemas andino xéricos, en especial los aún existentes entre los distritos de Limatambo y Curahuasi, pertenecientes a la cuenca del Apurímac, han sido deteriorados y destruidos debido al crecimiento urbano desordenado, la expansión de tierras agrícolas, contaminación, procesos erosivos y deterioro ambiental provocando como consecuencia la pérdida de la diversidad biológica de la zona en estudio, que a pesar de contar con adaptaciones especializadas para soportar escasez de agua, frente a los problemas ambientales mencionados y las actividades antrópicas como la construcción de obras civiles sin ningún estudio de impacto ambiental previo, aún se mantienen raleadas en estado de relictos boscosos por disección y desaparición de hábitats.

\section{HIPÓTESIS}

La cuenca del Apurimac: Limatambo - Cúnyaq y Curahuasi, por sus condiciones climáticas y edáficas particulares, presenta una elevada fitodiversidad xeroficea.

\section{ObJETIVO GENERAL}

Determinar la diversidad florística de la cuenca del Apurímac: Limatambo - Cúnyaq y Curahuasi a través de la gradiente altitudinal.

\section{EsPecíficos}

1. Evaluar la flora xerofitica del área en estudio y su fenología.

2. Realizar el análisis comparativo de los parámetros poblacionales: diversidad, similitud, abundancia y dominancia en ambos márgenes de la cuenca.

3. Determinar las especies endémicas del área de trabajo.

\section{MÉtodos De GAMPo}

El método desarrollado en el presente trabajo de investigación es evaluativo y analitico. Para determinar la flora xerofitica se empleó el método de transecto, a través de la gradiente altitudinal desde el piso de valle en ambas márgenes y para el estudio de la fitodiversidad, en ambos flancos, se utilizó el análisis comparativo, empleando parámetros estadísticos como los de Shanon - Winner y Jaccard.

\section{MÉtodo DE GABINETE}

Las determinaciones sistemáticas se realizaron en base a comparaciones y descripciones en el Herbario Vargas (Cuz) y consulta de bibliografia especializada.

\section{DETERMINACIÓN DE LA DIVERSIDAD DE ESPECIES}

Una comunidad es más compleja mientras mayor sea el número de especies que la compongan, proporcionando más vías de flujo de energía en la cadena trófica y mientras menos dominancia presente con una o pocas especies con respecto a las demás.

A las características de las comunidades que miden ese grado de complejidad, se le llama diversidad; aun cuando la diversidad es un concepto que pueda entenderse fácilmente en forma cualitativa, la expresión cuantitativa de ésta es aún confusa. Existe una gran cantidad de índices que estiman la diversidad de una comunidad; de estos los que se basan en la Teoría de la Información son los que más impulso han tenido, a pesar de sus limitaciones (Matteucci y Colma, 1982).

\section{Índice de diversidad de Shannon Winner}

Este índice toma en cuenta los dos componentes de la diversidad, número de especies y equidad, como la uniformidad de la distribución del número de individuos de cada especie; de acuerdo con esto, un mayor número de especie incrementa la diversidad y además una mayor uniformidad también lo hará utilizando la siguiente fórmula:

$$
\mathrm{H}^{\prime}=\operatorname{piLog} 2 \mathrm{Pi} \sum
$$

Donde:

$$
\begin{aligned}
\mathrm{H}= & \text { Diversidad (bits/individuos) } \\
\mathrm{Pi}= & \text { Proporción de números de individuos de } \\
& \text { la especie i, con respecto al total (ni / Nt) }
\end{aligned}
$$

\section{Indice de Simpson}

El índice de Simpson es otro método utilizado comúnmente para determinar la diversidad de una comunidad vegetal. Este índice considera no sólo el número de especies (S) y el número total de individuos $(\mathrm{N})$, sino también la proporción del total que ocurre en cada especie. Para calcular el índice de forma apropiada, se utiliza la siguiente fórmula:

$$
\mathrm{S}=1 /\left(\mathrm{n}\left(\mathrm{n}_{\mathrm{i}}-1\right) / \mathrm{N} \sum(\mathrm{N}-1)\right.
$$

Donde:

S= Índice de Simpson

$\mathrm{ni}=$ Número de individuos en la misma especie

$\mathrm{N}=$ Número total de individuos en la comunidad 


\section{Resultados}

De las colecciones realizadas en el área de estudio se han encontrado las siguientes

especies que se presentan por familias en orden alfabético.

\section{Agavaceae}

Agrale cordilletensis 7. Lade \& G. Pino

Furcraea andina Trelease

Furcrata occidentalis Trelease

\begin{tabular}{l}
\hline Alstromeriaceae \\
Bomarea ozala (Carmilles) Mirbel \\
\hline Amaranthaceae \\
Amoranthus hybridus Linnaets \\
Comphrena degans C. Mortius \\
Grilleminea densa (Willdnow) Moguin \\
Iresine diffusa Humb \& Bonpl. ex \\
Willdenow
\end{tabular}

\section{Equisetaceae}

Equisetam bogolense H. B. K.

\section{Escalloniaceae \\ Escallonia henerae Molffeld}

\section{Euphorbiaceae}

Cnidoscolus perwoianus (Muell. Arg) Pax \& Hoffman

Dalechampia arislolocaefolia H.B.K.

Jatropha augustï $P a x$ \&ै Hoffman

Marihot anomala Pohl sub sp panomiana (Muell. Arg) Reger \& Appan

Ricinus conmunis Linnaeus

\begin{tabular}{|c|c|}
\hline Nacardiaceae & Fabaceae \\
\hline Schinus molle Linnaeus & 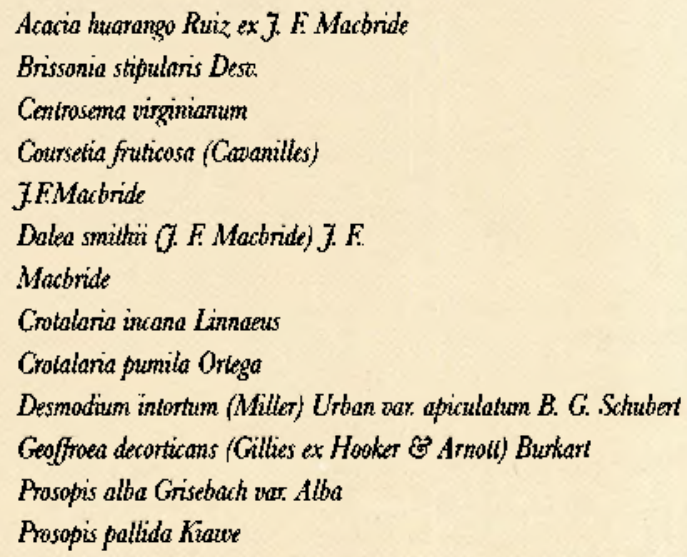 \\
\hline $\begin{array}{l}\text { Annonaceae } \\
\text { Annona chirmola Mill }\end{array}$ & $\begin{array}{l}\text { Iridaceae } \\
\text { Hesperoxiphion peravianum (Baker) } \\
\text { Baker }\end{array}$ \\
\hline $\begin{array}{l}\text { Apiaceae } \\
\text { Hydrocotyle bonariensis Lam, Synonyms }\end{array}$ & $\begin{array}{l}\text { Lamiaceae } \\
\text { Hyptis pectinata (L) Poiret } \\
\text { Minthosiachys glabrescens } \\
\text { Sadvia malacophylla Benth }\end{array}$ \\
\hline $\begin{array}{l}\text { Araliaceae } \\
\text { Pentapnnax angelicifolitus Griseb. }\end{array}$ & $\begin{array}{l}\text { Loasaceae } \\
\text { Menizelia fendleriana Urban \& Gilg. } \\
\text { Nasa poissoniana (Urb \& Gilg) Weigend }\end{array}$ \\
\hline $\begin{array}{l}\text { Asclepiadaceae } \\
\text { Melimia penwiana Schll }\end{array}$ & $\begin{array}{l}\text { Malvaceae } \\
\text { Abutilon pentinomum (Lamarck) Keamev } \\
\text { Anoda cristata (L) Schlldl. }\end{array}$ \\
\hline 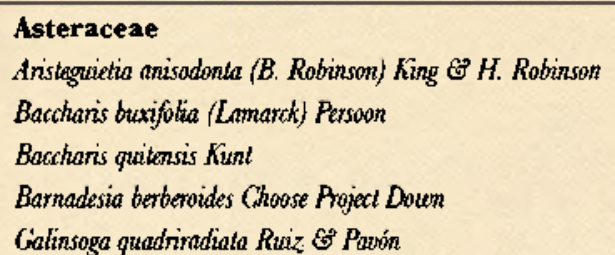 & $\begin{array}{l}\text { Nyctaginaceae } \\
\text { Boertaria diffusa Lennatus } \\
\text { Colignonia ovalifolia Heimen } \\
\text { Mirabilis elegans Cavanillea } \\
\text { Mirobilis prostrotn }(R . \& P) \text { Heimerl }\end{array}$ \\
\hline
\end{tabular}


Fenreyranthus uerbascifolius (H.B.K.) $H$.

Robinson \& Bretell

Pertis sessiliffora (Lessing) Sctudlz-Bip

Pluchea zamalloae (Cabrera) $H$.

Robinson \& Cuairecasas

Porophyllum ruderale (acquin) Cassini

Tessaria integrifolia Ruiz os Pavón

Trixis divaricata (H.B.K.) Sprengel

Vetnonia scorpioides (Lamarck) Persoon

Viguiera procumbens (Prrsoon) SE Blake

Vyuiera lanceolada Brillon.

Zimnia perwivana (L) Linnaeus

\section{Berberidaceae \\ Berderis humbertiana JF Macbride}

\section{Onagraceae}

Ludurigia peruviana (L) H. Hara

\section{Piperaceae}

Peperomia galioides H.B.K.

\section{Poaceae}

Agrostis nigricans ( $R$ \& P P) Poir

Aristida adscensionis Linndeus

Botriochloa saccharioides (Suarnz) Rydberg

Cenchrus echinatus Linnacus

Cenchrus myosuroides Humboldh,

Bomplant y Kumt

Dielisochloa saccharioides

Melica scabra Kinth.

Paspalum depauperatum 7.S. Presl

Paspalum ceresinides Carrillo

Penniselum bambusforme (Foumier

Hansly ex B. D. Jackson

Phragmithes australis (Cavanilles)

Trinius ex Steudel

Setaria panifora (poir) Kerguelen

\section{Bignoniaceae}

Tecoma arequitensis (Sprague)

Sandwith

Tecoma sambucifolia H.B.K.

Jacaranda acutifolia Humboldt

Bonpland.

\begin{tabular}{|c|c|}
\hline $\begin{array}{l}\text { Bombacaceae } \\
\text { Eriotheca vargasii (Cuatrecasas) Robyns }\end{array}$ & $\begin{array}{l}\text { Rosaceae } \\
\text { Rubus acanthophyllus Focke } \\
\text { Rubus unticifolus Poir }\end{array}$ \\
\hline $\begin{array}{l}\text { Boraginaceae } \\
\text { Heliotropitam incanum } R . \text { E P. }\end{array}$ & $\begin{array}{l}\text { Sapindaceae } \\
\text { Cardiaspermum corindun Linmnacus } \\
\text { Dodonaes viscosa Jacqin } \\
\text { Sapindus saponaria Linnaeus } \\
\text { Serjonia inflata Poeppig } \\
\text { Serjonia longistipula Radkofer }\end{array}$ \\
\hline $\begin{array}{l}\text { Bromeliaceae } \\
\text { Raya densiflora Haras } \\
\text { Puya longistyla (R E. P) LR. Smith } \\
\text { Tillandsia bryoides Grisebach ex Baker } \\
\text { Tillandsia capillaris } R \text {. \& P. fo. Capillaries } \\
\text { Tillandsia caulescens Brongniart ex } \\
\text { Baker }\end{array}$ & $\begin{array}{l}\text { Scrophulariaceae } \\
\text { Veronica anagallis-aquatica Linnaeus }\end{array}$ \\
\hline $\begin{array}{l}\text { Cactaceae } \\
\text { Brouningia vindis (Rauh \& Backeberg) F Buxbaum } \\
\text { Chistocactus brewispinus }\end{array}$ & $\begin{array}{l}\text { Solanaceae } \\
\text { Nicotiana glauca Graham } \\
\text { Nicotiana tomentosa Ruiz \& Proon }\end{array}$ \\
\hline
\end{tabular}

\section{Rhamnaceae}

Zixiphus mistol Griseback 
100 AÑos

Echinopsis maximiliana Heyder

Opuntia ficus-indica (L) Millar

Opuntia soehrensï (Bnitton \& Rose) Hunt

Lif $T$

Weberbateroceraus euzcoensis Knize

\begin{tabular}{|c|c|}
\hline $\begin{array}{l}\text { Caricaceae } \\
\text { Canica querifolia (A.St.Hilaite) } \\
\text { Hieronymus }\end{array}$ & $\begin{array}{l}\text { Tiphaceae } \\
\text { Typha dominguensis Pers }\end{array}$ \\
\hline $\begin{array}{l}\text { Commelinaceae } \\
\text { Commelina longicoutis } R . \& P .\end{array}$ & $\begin{array}{l}\text { Ulmaceae } \\
\text { Trema micrantha (L) Blume. }\end{array}$ \\
\hline $\begin{array}{l}\text { Convolvulaceae } \\
\text { Ipomoea tarnea Jacquin } \\
\text { Ipomoea nil (L) Roth } \\
\text { Jaquemontia branthetii Moritand }\end{array}$ & $\begin{array}{l}\text { Velloziaceae } \\
\text { Vargaceniopsis vargasii (lyman B. Smith) Lyman B. Smith }\end{array}$ \\
\hline $\begin{array}{l}\text { Cucorbitaceae } \\
\text { Cucumis dipsacets C. G. Ehrenh. Ex Spach. }\end{array}$ & $\begin{array}{l}\text { Verbenaceae } \\
\text { Aloysia scorodonioides (H.B.K.) } \\
\text { Chamisso } \\
\text { Lanlana canara Linnoeus } \\
\text { Lantana canescens H.B.K } \\
\text { Lanlana gitunosa Poeppig } \\
\text { Lantana urticaffolia Mill }\end{array}$ \\
\hline $\begin{array}{l}\text { Cyperaceae } \\
\text { Cpperus hermaphroditus (Gacquin) } \\
\text { Silandey uar, hermaphroditus }\end{array}$ & $\begin{array}{l}\text { Zigophyllaceae } \\
\text { Kallstracmia parofifora Norton }\end{array}$ \\
\hline
\end{tabular}

\section{Physatis pentiana Linnaeus}

Solanan saponaceum Dunal

Gráfico $\mathbf{N}^{\circ}$ 1: Números de especies en relación a las familias existentes en el Área de estudio.

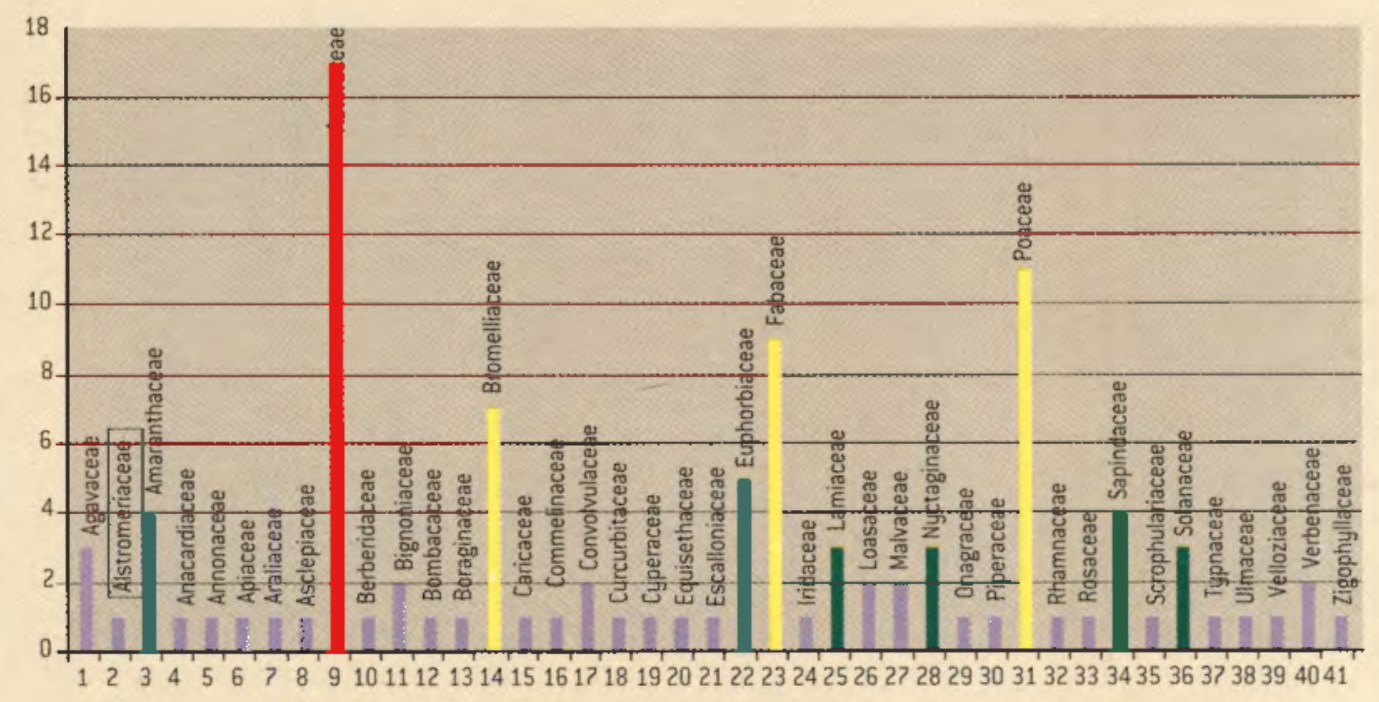


2. De las variables poblacionales y del valor de importancia

Las siguientes tablas muestran los diferentes parámetros poblacionales como Frecuencia $(\mathbf{F})$, Frecuencia relativa (Fr), Densidad (D), Densidad relativa (Dr), Cobertura $(\mathrm{C})$, Cobertura relativa
(Cr), y Valor de Importancia para las 05 parcelas en el área de estudio.

El cuadro I muestra que la especie Opuntia ficus indica es la especie dominante.

El cuadro 2, revela que la especie Furcraea andina es la especie que presenta mayor índice de

Primer Transecto: Wayronq'a, Altitud 2850 m, Área: 50 x 5 m

Cuadro $\mathbf{N}^{\circ} 1$ : Variables poblacionales para la parcela 01 .

\begin{tabular}{|c|c|c|c|c|c|c|c|c|c|}
\hline Especies & $\mathbf{N}^{\circ}$ Ind. & Ocur & $\mathbf{F}$ & $\mathbf{F r}$ & $\mathbf{D}$ & Dr & Do & Dor & IVI \\
\hline Opuntia ficus - indica & 182 & 5 & 100 & 6,85 & 0,728 & 22,09 & 100,00 & 6,85 & 35,79 \\
\hline Tecoma sambucifolia & 4 & 1 & 20 & 1,37 & 0,016 & 0,49 & 20,00 & 1,37 & 3,23 \\
\hline Schinus molle & 5 & 1 & 20 & 1,37 & 0,02 & 0,61 & 20,00 & 1,37 & 3,35 \\
\hline Mirabilis prostrata & 8 & 2 & 40 & 2,74 & 0,032 & 0,97 & 40,00 & 2,74 & 6,45 \\
\hline Ophryosporus peruviana & 18 & 2 & 40 & 2,74 & 0,072 & 2,18 & 40,00 & 2,74 & 7,66 \\
\hline Minthostachys glabrescens & 3 & 1 & 20 & 1,37 & 0.012 & 0,36 & 20,00 & 1,37 & 3,10 \\
\hline Trixis divaricada & 14 & 2 & 40 & 2,74 & 0,056 & 1,70 & 40,00 & 2,74 & 7,18 \\
\hline Commelina longicaulis & 16 & 2 & 40 & 2,74 & 0,064 & 1,94 & 40,00 & 2,74 & 7,42 \\
\hline Dodonaea viscosa & 13 & 3 & 60 & 4,11 & 0,052 & 1,58 & 60,00 & 4,11 & 9,80 \\
\hline Serjania inflata & 31 & 4 & 80 & 5,48 & 0,124 & 3,76 & 80,00 & 5,48 & 14,72 \\
\hline Lantana glutinosa & 25 & 2 & 40 & 2,74 & 0,1 & 3,03 & 40,00 & 2,74 & 8,51 \\
\hline Galinsoga quadriradiata & 68 & 5 & 100 & 6,85 & 0,272 & 8,25 & 100,00 & 6,85 & 21,95 \\
\hline Desmodium uncinatum & 14 & 2 & 40 & 2,74 & 0,056 & 1,70 & 40,00 & 2,74 & 7,18 \\
\hline Iresine difusa & 12 & 1 & 20 & 1,37 & 0,048 & 1,46 & 20,00 & 1,37 & 4,20 \\
\hline Gomphrena elegans & 15 & 2 & 40 & 2,74 & 0,06 & 1,82 & 40,00 & 2,74 & 7,30 \\
\hline Nasa poissoniana & 18 & 2 & 40 & 2,74 & 0,072 & 2,18 & 40,00 & 2,74 & 7,66 \\
\hline Mentzelia fendleriana & 11 & 1 & 20 & 1,37 & 0,044 & 1,33 & 20,00 & 1,37 & 4,07 \\
\hline Pennisetum bambusiforme & 58 & 3 & 60 & 4,11 & 0,232 & 7,04 & 60,00 & 4,11 & 15,26 \\
\hline Abutilom peruvianum & 9 & 1 & 20 & 1,37 & 0,036 & 1,09 & 20,00 & 1,37 & 3,83 \\
\hline Colignonia ovalifolia & 11 & 1 & 20 & 1,37 & 0,044 & 1,33 & 20,00 & 1,37 & 4,07 \\
\hline Cardiospermum corindum & 47 & 2 & 40 & 2,74 & 0,188 & 5,70 & 40,00 & 2,74 & 11,18 \\
\hline Melinia peruviana & 13 & 2 & 40 & 2,74 & 0,052 & 1,58 & 40,00 & 2,74 & 7,06 \\
\hline Crotalaria incana & 5 & 1 & 20 & 1,37 & 0,02 & 0,61 & 20,00 & 1,37 & 3,35 \\
\hline Tecoma arequipensis & 14 & 1 & 20 & 1,37 & 0,056 & 1,70 & 20,00 & 1,37 & 4,44 \\
\hline Pluchea zamalloae & 23 & 2 & 40 & 2,74 & 0,092 & 2,79 & 40,00 & 2,74 & 8,27 \\
\hline Berberis humbertiana & 2 & 1 & 20 & 1,37 & 0,008 & 0,24 & 20,00 & 1,37 & 2,98 \\
\hline Escallonia herrerae & 4 & 1 & 20 & 1,37 & 0,016 & 0,49 & 20,00 & 1,37 & 3,23 \\
\hline Aristeguietia anisodonta & 9 & 2 & 40 & 2,74 & 0,036 & 1,09 & 40,00 & 2,74 & 6,57 \\
\hline Baccharis buxifolia & 35 & 3 & 60 & 4,11 & 0,14 & 4,25 & 60,00 & 4,11 & 12,47 \\
\hline Amaranthus hybridus & 20 & 2 & 40 & 2,74 & 0,08 & 2,43 & 40,00 & 2,74 & 7,91 \\
\hline Bomarea ovata & 4 & 1 & 20 & 1,37 & 0,016 & 0,49 & 20,00 & 1,37 & 3,23 \\
\hline Baccharis quitensis & 6 & 1 & 20 & 1,37 & 0,024 & 0,73 & 20,00 & 1,37 & 3,47 \\
\hline Flourencia polisépala & 23 & 2 & 40 & 2,74 & 0,092 & 2,79 & 40,00 & 2,74 & 8,27 \\
\hline Tillandsia bryoides & 15 & 1 & 20 & 1,37 & 0,06 & 1,82 & 20,00 & 1,37 & 4,56 \\
\hline Tillandsia capillaris & 27 & 3 & 60 & 4,11 & 0,108 & 3,28 & 60,00 & 4,11 & 11,50 \\
\hline Brissonia stipularis & 13 & 1 & 20 & 1,37 & 0,052 & 1,58 & 20,00 & 1,37 & 4,32 \\
\hline Hyptis pectinata & 4 & 1 & 20 & 1,37 & 0,016 & 0,49 & 20,00 & 1,37 & 3,23 \\
\hline Anoda cristata & 12 & 1 & 20 & 1,37 & 0,048 & 1,46 & 20,00 & 1,37 & 4,20 \\
\hline Peperomia galioides & 8 & 1 & 20 & 1,37 & 0,032 & 0,97 & 20,00 & 1,37 & 3,71 \\
\hline \multirow[t]{2}{*}{ Rubus acanthophyllus } & 5 & 1 & 20 & 1,37 & 0,02 & 0,61 & 20,00 & 1,37 & 3,35 \\
\hline & & & 1460 & 100,00 & 3,296 & 100,00 & 1460 & 100,00 & \\
\hline
\end{tabular}


Segundo Transecto: Sauceda, Altitud 2420 m, Área; 50 x 5 m

Cuadro $\mathbf{N}^{\circ}$ 2: Variables poblacionales para la parcela 02.

\begin{tabular}{|c|c|c|c|c|c|c|c|c|c|}
\hline Especies & $\mathbf{N}^{\circ}$ Ind. & Ocur & $\mathbf{F}$ & $\mathbf{F r}$ & $\mathbf{D}$ & Dr & Do & Der & IVI \\
\hline Pentapanax angelicifolius & 6 & 1 & 20 & 1,23 & 0,024 & 0,78 & 20 & 1,23 & 3,25 \\
\hline Furcraea andina & 79 & 5 & 100 & 6,17 & 0,316 & 10,26 & 100 & 6,17 & 22,61 \\
\hline Cleistocactus brevispinus & 3 & 1 & 20 & 1,23 & 0,012 & 0,39 & 20 & 1,23 & 2,86 \\
\hline Vargaseniopsis vargasii & 34 & 4 & 80 & 4,94 & 0,136 & 4,42 & 80 & 4,94 & 14,29 \\
\hline Eriotheca vargasii & 3 & 1 & 20 & 1,23 & 0,012 & 0,39 & 20 & 1,23 & 2,86 \\
\hline Acacia huarango & 17 & 2 & 40 & 2,47 & 0,068 & 2,21 & 40 & 2,47 & 7,15 \\
\hline Cardiospermum corindum & 2 & 1 & 20 & 1,23 & 0,008 & 0,26 & 20 & 1,23 & 2,73 \\
\hline Jacquemontia parvifolia & 3 & 1 & 20 & 1,23 & 0,012 & 0,39 & 20 & 1,23 & 2,86 \\
\hline Puya longistyla & 12 & 2 & 40 & 2,47 & 0,048 & 1,56 & 40 & 2,47 & 6,50 \\
\hline Lantana camara & 11 & 2 & 40 & 2,47 & 0,044 & 1,43 & 40 & 2,47 & 6,37 \\
\hline Echinopsis Maximiliano & 2 & 1 & 20 & 1,23 & 0,008 & 0,26 & 20 & 1,23 & 2,73 \\
\hline Siguiera procumbens & 5 & 1 & 20 & 1,23 & 0,02 & 0,65 & 20 & 1,23 & 3,12 \\
\hline Lantana canescens & 7 & 1 & 20 & 1,23 & 0,028 & 0,91 & 20 & 1,23 & 3,38 \\
\hline Solanum saponaceum & 20 & 3 & 60 & 3,70 & 0,08 & 2,60 & 60 & 3,70 & 10,00 \\
\hline Trixis divaricada & 12 & 2 & 40 & 2,47 & 0,048 & 1,56 & 40 & 2,47 & 6,50 \\
\hline Aloysia scorodonioides & 4 & 1 & 20 & 1,23 & 0,016 & 0,52 & 20 & 1,23 & 2,99 \\
\hline Coursetia fruticosa & 2 & 1 & 20 & 1,23 & 0,008 & 0,26 & 20 & 1,23 & 2,73 \\
\hline Tillandsia caulescens & 6 & 1 & 20 & 1,23 & 0,024 & 0,78 & 20 & 1,23 & 3,25 \\
\hline Mentzelia fendleriana & 1 & 1 & 20 & 1,23 & 0,004 & 0,13 & 20 & 1,23 & 2,60 \\
\hline Escallonia herrerae & 1 & 1 & 20 & 1,23 & 0,004 & 0,13 & 20 & 1,23 & 2,60 \\
\hline Tessaria integrifolia & 68 & 4 & 80 & 4,94 & 0,272 & 8,83 & 80 & 4,94 & 18,71 \\
\hline Typha dominguensis & 74 & 4 & 80 & 4,94 & 0,296 & 9,61 & 80 & 4,94 & 19,49 \\
\hline Ludwigia peruviana & 47 & 4 & 80 & 4,94 & 0,188 & 6,10 & 80 & 4,94 & 15,98 \\
\hline Hydrocotyle bonariensis & 56 & 3 & 60 & 3,70 & 0,224 & 7,27 & 60 & 3,70 & 14,68 \\
\hline Pragmithes australis & 78 & 2 & 40 & 2,47 & 0,312 & 10,13 & 40 & 2,47 & 15,07 \\
\hline Equisetum bogotense & 43 & 5 & 100 & 6,17 & 0,172 & 5,58 & 100 & 6,17 & 17,93 \\
\hline Salvia malacophylla & 6 & 1 & 20 & 1,23 & 0,024 & 0,78 & 20 & 1,23 & 3,25 \\
\hline Trema micrantha & 11 & 2 & 40 & 2,47 & 0,044 & 1,43 & 40 & 2,47 & 6,37 \\
\hline Baccharis buxifolia & 17 & 2 & 40 & 2,47 & 0,068 & 2,21 & 40 & 2,47 & 7,15 \\
\hline Barnadesia berberoides & 5 & 1 & 20 & 1,23 & 0,02 & 0,65 & 20 & 1,23 & 3,12 \\
\hline Jacaranda acutifolia & 4 & 1 & 20 & 1,23 & 0,016 & 0,52 & 20 & 1,23 & 2,99 \\
\hline Pectis sessiliiflora & 22 & 3 & 60 & 3,70 & 0,088 & 2,86 & 60 & 3,70 & 10,26 \\
\hline Cyperus hermafroditas & 45 & 4 & 80 & 4,94 & 0,18 & 5,84 & 80 & 4,94 & 15,72 \\
\hline Flaveria bidentis & 19 & 3 & 60 & 3,70 & 0,076 & 2,47 & 60 & 3,70 & 9,87 \\
\hline Vernonia scorpioides & 13 & 2 & 40 & 2,47 & 0,052 & 1,69 & 40 & 2,47 & 6,63 \\
\hline Carica quercifolia & 3 & 1 & 20 & 1,23 & 0,012 & 0,39 & 20 & 1,23 & 2,86 \\
\hline Manihot anomala & 12 & 2 & 40 & 2,47 & 0,048 & 1,56 & 40 & 2,47 & 6,50 \\
\hline Ipomoea carnea & 7 & 1 & 20 & 1,23 & 0,028 & 0,91 & 20 & 1,23 & 3,38 \\
\hline Veronica anagallis-aquatica & 10 & 3 & 60 & 3,70 & 0,04 & 1,30 & 60 & 3,70 & 8,71 \\
\hline & & & 1620 & 100,00 & 3,08 & 100,00 & 1620 & 100,00 & \\
\hline
\end{tabular}

importancia, desde el punto de vista ecológico, seguida de Tipha dominguensis y Tessaria integrifolia.

El cuadro 3 muestra a la especie Guillenminea densa como la especie con mayor índice de importancia, seguida de Opuntia soerhensii y Geoffroea decorticans, las cuales muestran una formación vegetal que corresponde a un bosque seco.
El cuadro 4 muestra las variables poblacionales para la localidad del Puente de Cúnyaq, la especie Paspalum ceresioides es la especie que presenta mayor índice de importancia para esta localidad, seguida de Bothriochloa saccharoides, especies propias de un lugar con una sucesión vegetal considerable. 
Tercer Transecto: Wayna Masa, Altitud $2200 \mathrm{~m}$, Área: 50 x $5 \mathrm{~m}$

Cuadro $N^{\circ} 3$ : Variables poblacionales para la parcela 03 .

\begin{tabular}{|c|c|c|c|c|c|c|c|c|c|}
\hline Especies & $\mathbf{N}^{\circ}$ Ind. & Ocur & $\mathbf{F}$ & $\mathbf{F r}$ & $\mathbf{D}$ & Dr & D & Dor & IVI \\
\hline Mirabilis elegans & 6 & 1 & 20 & 1,56 & 0,024 & 1,41 & 20 & 1,56 & 4,53 \\
\hline Kallstroemia parviflora & 12 & 2 & 40 & 3,13 & 0,048 & 2,81 & 40 & 3,13 & 9,06 \\
\hline Physalis peruviana & 2 & 1 & 20 & 1,56 & 0,008 & 0,47 & 20 & 1,56 & 3,59 \\
\hline Prosopis pallida & 23 & 4 & 80 & 6,25 & 0,092 & 5,39 & 80 & 6,25 & 17,89 \\
\hline Acacia huarango & 21 & 4 & 80 & 6,25 & 0,084 & 4,92 & 80 & 6,25 & 17,42 \\
\hline Guilleminea densa & 38 & 5 & 100 & 7,81 & 0,152 & 8,90 & 100 & 7,81 & 24,52 \\
\hline Opuntia soehrensii & 34 & 5 & 100 & 7,81 & 0,136 & 7,96 & 100 & 7,81 & 23,59 \\
\hline Agrostis nigricans & 16 & 2 & 40 & 3,13 & 0,064 & 3,75 & 40 & 3,13 & 10,00 \\
\hline Ziziphus mistol & 19 & 3 & 60 & 4,69 & 0,076 & 4,45 & 60 & 4,69 & 13,82 \\
\hline Dalechampia aristolochiaefolia & 9 & 2 & 40 & 3,13 & 0,036 & 2,11 & 40 & 3,13 & 8,36 \\
\hline Opuntia Picus-indica & 22 & 4 & 80 & 6,25 & 0,088 & 5,15 & 80 & 6,25 & 17,65 \\
\hline Heliotropium incarum & 7 & 1 & 20 & 1,56 & 0,028 & 1,64 & 20 & 1,56 & 4,76 \\
\hline Browningia viridis & 2 & 1 & 20 & 1,56 & 0,008 & 0,47 & 20 & 1,56 & 3,59 \\
\hline Serjania longistipula & 12 & 2 & 40 & 3,13 & 0,048 & 2,81 & 40 & 3,13 & 9,06 \\
\hline Prosopis alba & 8 & 2 & 40 & 3,13 & 0,032 & 1,87 & 40 & 3,13 & 8,12 \\
\hline Boerhavia difusa & 18 & 3 & 60 & 4,69 & 0,072 & 4,22 & 60 & 4,69 & 13,59 \\
\hline Geoffroea decorticans & 47 & 4 & 80 & 6,25 & 0,188 & 11,01 & 80 & 6,25 & 23,51 \\
\hline Anoda cristata & 23 & 3 & 60 & 4,69 & 0,092 & 5,39 & 60 & 4,69 & 14,76 \\
\hline Pentapanax angelisifolius & 8 & 1 & 20 & 1,56 & 0,032 & 1,87 & 20 & 1,56 & 5,00 \\
\hline Centrosema virginianum & 7 & 1 & 20 & 1,56 & 0,028 & 1,64 & 20 & 1,56 & 4,76 \\
\hline Crotalaria pumila & 5 & 1 & 20 & 1,56 & 0,02 & 1,17 & 20 & 1,56 & 4,30 \\
\hline Nicotiana tomentosa & 13 & 3 & 60 & 4,69 & 0,052 & 3,04 & 60 & 4,69 & 12,42 \\
\hline Annona chirimola & 3 & 1 & 20 & 1,56 & 0,012 & 0,70 & 20 & 1,56 & 3,83 \\
\hline Zinnia peruviana & 29 & 2 & 40 & 3,13 & 0,116 & 6,79 & 40 & 3,13 & 13,04 \\
\hline Manihot anomala & 17 & 2 & 40 & 3,13 & 0,068 & 3,98 & 40 & 3,13 & 10,23 \\
\hline Aristida adscensionis & 15 & 2 & 40 & 3,13 & 0,06 & 3,51 & 40 & 3,13 & 9,76 \\
\hline Rubus urticifolius & 3 & 1 & 20 & 1,56 & 0,012 & 0,70 & 20 & 1,56 & 3,83 \\
\hline \multirow[t]{2}{*}{ Nicotiana glauca } & 8 & 1 & 20 & 1,56 & 0,032 & 1,87 & 20 & 1,56 & 5,00 \\
\hline & & & 1280 & 100,00 & 1,708 & 100,00 & 1280 & 100,00 & \\
\hline
\end{tabular}

El cuadro 5 muestra las variables poblacionales para la localidad de Q'onoq. La especie herbácea Vargaseniopsis vargasii es la que presenta mayor índice de importancia, seguida de Setaria parviflora y Dielsiochloa saccharioides.

\section{De los índices de diversidad y dominancia}

Utilizando Past program se han obtenido los siguientes índices, de los cuales se hará la interpretación del índice de diversidad de Shannon, de Simpson, Menhinick, Margalef, el de Berger Parker, el de dominancia.

De acuerdo con el cuadro 6 , los diferentes índices de diversidad muestran que la localidad de Wayronqa es la más diversa entre las localidades estudiadas, con un índice de Shannon de 3,689 , seguido por la localidad de Sauceda. Las localidades de Huayna Masa y Qonoq presentan el mismo índice con una diversidad relativamente alta, mostrando un valor de 3,332, y finalmente la localidad de Puente Cúnyaq con el más bajo valor de 3,091 de igual manera con los demás índices.

\section{De la similitud de localidades}

Usando también Past Program con el Indice de Jactar se tiene que las comunidades de Puente Cúnyaq y Q'onoq son las más similares en la composición vegetal, las demás son diferentes entre sí.

\section{Especies endémicas de la zona de interés}

- En la cuenca del Apurimac se encuentraran especies representativas de gran interés; entre las más importantes se tiene:

- Pentapanax angelisisolius, "q'ello p'ati", especie en proceso de extinción. 
Cuarto Transecto, Localidad: Puente Cúnyaq, Altitud 1980 m, Área: 50 x 5 m.

Cuadro $\mathbf{N}^{\circ}$ 4: Variables poblacionales para la parcela 04.

\begin{tabular}{|c|c|c|c|c|c|c|c|c|c|}
\hline Especies & $\mathbf{N}^{\circ}$ Ind. & Ocur & $\mathbf{F}$ & $\mathbf{F r}$ & $\mathbf{D}$ & Dr & $\mathbf{D}$ & Dor & IVI \\
\hline Cucumis dipsaceus & 18 & 1 & 20 & 1,69 & 0,072 & 4,55 & 20 & 1,69 & 7,94 \\
\hline Nasa poissoniana & 23 & 2 & 40 & 3,39 & 0,092 & 5,81 & 40 & 3,39 & 12,59 \\
\hline Onoseris gnaphalioides & 9 & 1 & 20 & 1,69 & 0,036 & 2,27 & 20 & 1,69 & 5,66 \\
\hline Viguiera lanceolada & 13 & 2 & 40 & 3,39 & 0,052 & 3,28 & 40 & 3,39 & 10,06 \\
\hline Opuntia soehrensii & 16 & 3 & 60 & 5,08 & 0,064 & 4,04 & 60 & 5,08 & 14,21 \\
\hline Acacia huarango & 7 & 2 & 40 & 3,39 & 0,028 & 1,77 & 40 & 3,39 & 8,55 \\
\hline Browningia viridis & 8 & 2 & 40 & 3,39 & 0,032 & 2,02 & 40 & 3,39 & 8,80 \\
\hline Apuntia Picus-indica & 12 & 1 & 20 & 1,69 & 0,048 & 3,03 & 20 & 1,69 & 6,42 \\
\hline Dalechampia aristolochiafolia & 15 & 2 & 40 & 3,39 & 0,06 & 3,79 & 40 & 3,39 & 10,57 \\
\hline Cnidoscolus peruvianus & 23 & 3 & 60 & 5,08 & 0,092 & 5,81 & 60 & 5,08 & 15,98 \\
\hline Vargaseniopsis vargasii & 17 & 3 & 60 & 5,08 & 0,068 & 4,29 & 60 & 5,08 & 14,46 \\
\hline Eriotheca vargasii & 8 & 2 & 40 & 3,39 & 0,032 & 2,02 & 40 & 3,39 & 8,80 \\
\hline Melica scabra & 27 & 3 & 60 & 5,08 & 0,108 & 6,82 & 60 & 5,08 & 16,99 \\
\hline Jatropa augustii & 21 & 3 & 60 & 5,08 & 0,084 & 5,30 & 60 & 5,08 & 15,47 \\
\hline Weberbauerocereus cuzcoensis & 6 & 2 & 40 & 3,39 & 0,024 & 1,52 & 40 & 3,39 & 8,29 \\
\hline Cenchrus myosuroides & 5 & 2 & 40 & 3,39 & 0,02 & 1,26 & 40 & 3,39 & 8,04 \\
\hline Ricinus conmunis & 15 & 4 & 80 & 6,78 & 0,06 & 3,79 & 80 & 6,78 & 17,35 \\
\hline Paspalum ceresioides & 69 & 5 & 100 & 8,47 & 0,276 & 17,42 & 100 & 8,47 & 34,37 \\
\hline Bothriochloa saccharoides & 47 & 5 & 100 & 8,47 & 0,188 & 11,87 & 100 & 8,47 & 28,82 \\
\hline Furcrae occidentales & 17 & 4 & 80 & 6,78 & 0,068 & 4,29 & 80 & 6,78 & 17,85 \\
\hline Puya dernsiflora & 11 & 3 & 60 & 5,08 & 0,044 & 2,78 & 60 & 5,08 & 12,95 \\
\hline \multirow[t]{2}{*}{ Agave cordillerensis } & 9 & 4 & 80 & 6,78 & 0,036 & 2,27 & 80 & 6,78 & 15,83 \\
\hline & & & 1180 & 100,00 & 1,584 & 100,00 & 1180 & 100,00 & \\
\hline
\end{tabular}

- Prosopis pallida, Prosopis alba y Geoffroea decorticans, especies maderables de interés en la ebanistería y frutos comestibles.

- Manihot anómala, "monte yuca", yuca silvestre de interés genético.

- Dalechampia aristochaefolia, "bella abanquina", ornamental, con brácteas vistosas.

- Ziziphus mistol, "asafray" o palo negro, de madera fina y frutos comestibles.

- Cnidoscolus peruvianus y jatropa augustii, conocidos como "wanarpo", plantas afrodisiacas.

- Eriotheca vargasii, "p'ati", planta eminentemente xerofitica que florece en la estación desfavorable, mayo a agosto, porque tiene estructuras subterráneas reservantes de agua.

- Weberbauerocereus cuzcoensis, "machu hawaq'ollay", es el gigantón más grande de la zona.

- Tecoma sambucifolia, "waranway", utilizado para elaborar muebles.

- Furcraea oxidentalis, "paqpa", planta fibrosa utilizada para la elaboración de cordeles y sogas, y el escapo para las construcciones.
- Vargaceniopsis vargasii, especie arrocetada y postrada, indicadora de suelos rocosos y xéricos.

\section{Conclusiones}

1. El estudio de la diversidad florística está representada por 42 familias botánicas, con 100 géneros y 122 especies y clara predominancia de Asteraceae y Poaceae, que coinciden con el número de especies de estas familias para los Andes del Perú.

2. En cuanto a la fenología, la gran mayoría de las especies estudiadas se reproducen (floración) de noviembre a marzo, con excepción de Eriotheca vargasii e Ipomoea carnea, cuya antesis floral es en los meses de junio a agosto.

3. De acuerdo con los resultados, las especies Opuntia ficus indica, Furcraea andina, Guilleminea densa, Paspalum ceresioides, y Vargaseniopsis vargasii, son las que presentan mayores índices de valor de importancia para las 5 parcelas establecidas. 
Quinto Transecto, Localidad: Q'onoq, Altitud $2150 \mathrm{~m}$, Área: $50 \times 5 \mathrm{~m}$.

Cuadro No 5: Variables problacionales para la parcela 05.

\begin{tabular}{|c|c|c|c|c|c|c|c|c|c|}
\hline Especies & $\mathbf{N}^{\circ}$ Ind. & Ocur & $\mathbf{F}$ & $\mathbf{F r}$ & D & Dr & D & Dor & IVI \\
\hline Tecoma arequipensis & 27 & 3 & 60 & 3,90 & 0,108 & 4,76 & 60 & 3,90 & 12,55 \\
\hline Pusya longistila & 22 & 3 & 60 & 3,90 & 0,088 & 3,88 & 60 & 3,90 & 11,67 \\
\hline Cnidoscolus peruvianus & 31 & 4 & 80 & 5,19 & 0,124 & 5,47 & 80 & 5,19 & 15,86 \\
\hline Viguiera procumbens & 8 & 2 & 40 & 2,60 & 0,032 & $1,4]$ & 40 & 2,60 & 6,61 \\
\hline Lantana urticaefolia & 3 & 1 & 20 & 1,30 & $0,0 \mid 2$ & 0,53 & 20 & 1,30 & 3,13 \\
\hline Vargaseniopsis vargasii & 120 & 5 & 100 & 6,49 & 0,48 & 21,16 & 100 & 6,49 & 34,15 \\
\hline Melica scabra & 5 & 1 & 20 & 1,30 & 0,02 & 0,88 & 20 & 1,30 & 3,48 \\
\hline Aloysia scorodonioides & 13 & 2 & 40 & 2,60 & 0,052 & 2,29 & 40 & 2,60 & 7,49 \\
\hline Ipomoea nil & 2 & ] & 20 & 1,30 & 0,008 & 0,35 & 20 & 1,30 & 2,95 \\
\hline Jaquemontia parvifolia & 13 & 3 & 60 & 3,90 & 0,052 & 2,29 & 60 & 3,90 & 10,08 \\
\hline Jatropa augustii & 5 & 1 & 20 & 1,30 & 0,02 & 0,88 & 20 & 1,30 & 3,48 \\
\hline Hesperoxiphion peruvianum & 9 & 2 & 40 & 2,60 & 0,036 & 1,59 & 40 & 2,60 & 6,78 \\
\hline Acacia huarango & 11 & 4 & 80 & 5,19 & 0,044 & 1,94 & 80 & 5,19 & 12,33 \\
\hline Eriotheca vargasii & 4 & 3 & 60 & 3,90 & 0,016 & 0,71 & 60 & 3,90 & 8,50 \\
\hline Dielsiochloa saccharioides & 38 & 5 & 100 & 6,49 & 0,152 & 6,70 & 100 & 6,49 & 19,69 \\
\hline Ferrcyranthus verbascifolius & 2 & 1 & 20 & 1,30 & 0,008 & 0,35 & 20 & 1,30 & 2,95 \\
\hline Andropogon saccharioides & 14 & 2 & 40 & 2,60 & 0,056 & 2,47 & 40 & 2,60 & 7,66 \\
\hline Porophyllum ruderale & 9 & 2 & 40 & 2,60 & 0,036 & 1,59 & 40 & 2,60 & 6,78 \\
\hline Cenchrus echinatus & 35 & 3 & 60 & 3,90 & 0,14 & 6,17 & 60 & 3,90 & 13,97 \\
\hline Dalea smithii & 25 & 4 & 80 & 5,19 & $0, \mathrm{l}$ & 4,41 & 80 & 5,19 & 14,80 \\
\hline Coursetia fruticosa & 30 & 4 & 80 & 5,19 & 0,12 & 5,29 & 80 & 5,19 & 15,68 \\
\hline Setaria parviftora & 40 & 5 & 100 & 6,49 & 0,16 & 7,05 & 100 & 6,49 & 20,04 \\
\hline Lantana canescens & 16 & 2 & 40 & 2,60 & 0,064 & 2,82 & 40 & 2,60 & 8,02 \\
\hline Weberbauerocereus cuzcoensis & 9 & 2 & 40 & 2,60 & 0,036 & 1,59 & 40 & 2,60 & 6,78 \\
\hline Paspalum depauperatum & 14 & 3 & 60 & 3,90 & 0,056 & 2,47 & 60 & 3,90 & 10,26 \\
\hline Kallstromia parviftora & 39 & 4 & 80 & 5,19 & 0,156 & 6,88 & 80 & 5,19 & 17,27 \\
\hline Browningia viridis & 3 & 1 & 20 & 1,30 & 0,012 & 0,53 & 20 & 1,30 & 3,13 \\
\hline Prosopis alba & 18 & 3 & 60 & 3,90 & 0,072 & 3,17 & 60 & 3,90 & 10,97 \\
\hline \multirow[t]{2}{*}{ Sapindus saponaria } & 2 & I & 20 & 1,30 & 0,008 & 0,35 & 20 & 1,30 & 2,95 \\
\hline & & & 1540 & 100,00 & 2,268 & 100,00 & 1540 & 100,00 & \\
\hline
\end{tabular}

Las especies endémicas son: Eriotheca vargasii, Ipomoea carnea, Zizipus mistol, Dalechampia anstolochaefolia, Jatropa augustii, IVeberbauerocereus cuzcoensis.

4. De los estudios realizados en el área se desprende que existen especies de interés biológico, económico y de interés ecológico. Están las adaptaciones al xerofitismo de Eniotheca vargasii que presentan estructuras xeromorfas subterráneas para la acumulación de agua, cuya utilización ocurre en la estación desfavorable.

Cuadro $\mathrm{N}^{\circ}$ 6: Indices de diversidad para las localidades en estudio.

\begin{tabular}{|c|c|c|c|c|c|}
\hline & Wayronqa & Sauceda & Huayna-Masa & Puente-Cúnyaq & Qonoq \\
\hline Taxa S & 40 & 39 & 28 & 22 & 28 \\
\hline Dominance D & 0,025 & 0,02564 & 0,03571 & 0,04545 & 0,03571 \\
\hline Shannon $\mathbf{H}$ & 3,689 & 3,664 & 3,332 & $3,09]$ & 3,332 \\
\hline Simpson (-D) & 0,975 & 0,9744 & 0,9643 & 0,9545 & 0,9643 \\
\hline Menhinick & 6,325 & 6,245 & 5,292 & 4,69 & 5,292 \\
\hline Margalef & 10,57 & 10,37 & 8,103 & 6,794 & 8,103 \\
\hline Berger-Parker & 0,025 & 0,02564 & $0,0357]$ & 0,04545 & $0,0357]$ \\
\hline
\end{tabular}


La presencia de las especies, Ziziphus mistol, Geoffroea decorticans, Prosopis alba son de interés económico por ser plantas maderables de frutos comestibles del bosque seco.

Desde el punto de vista biológico es importante la especie Pentapanax angelicifolius, propia de áreas xerofíticas, especie incluida en la lista roja del Instituto Nacional de Recursos Naturales (INRENA). En el aspecto cultural, la presencia de Jatropa augustii $y$ Cnidosculus peruvianus son de gran interés en la zona de estudio.

\section{ANEXo}

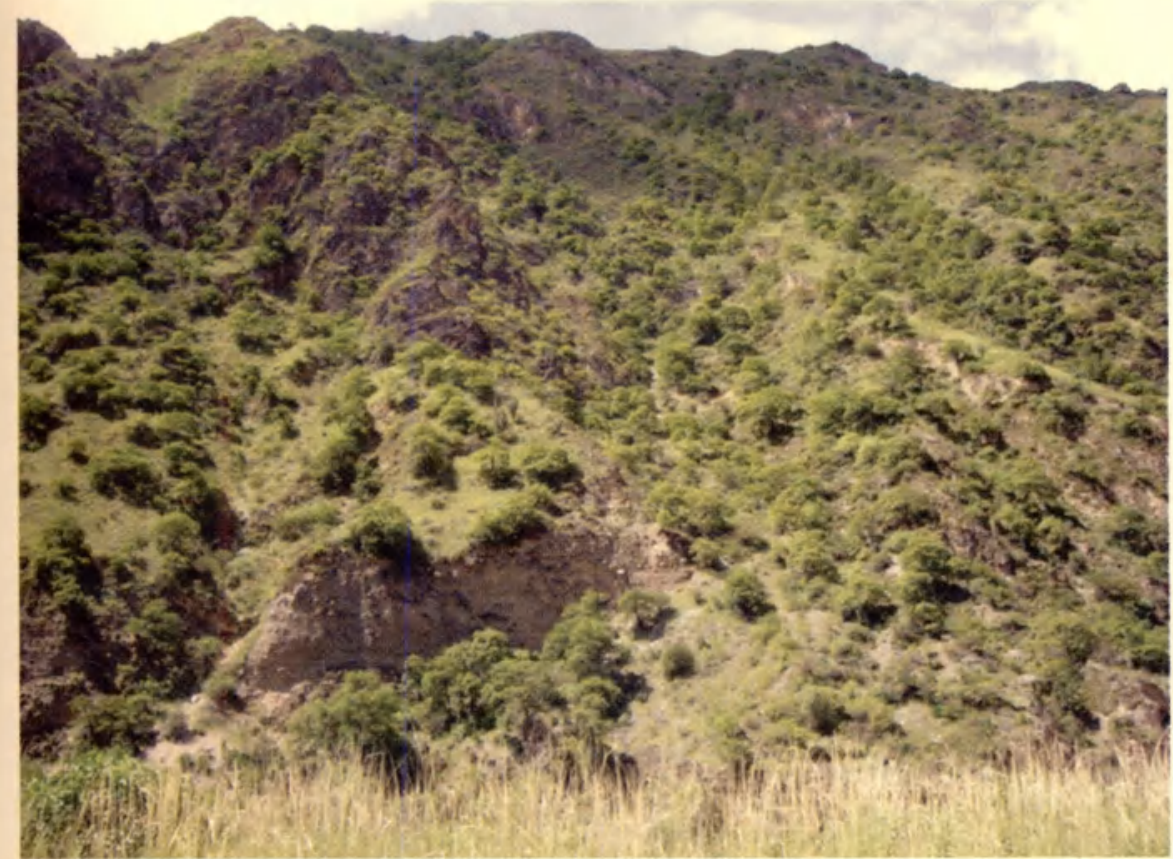

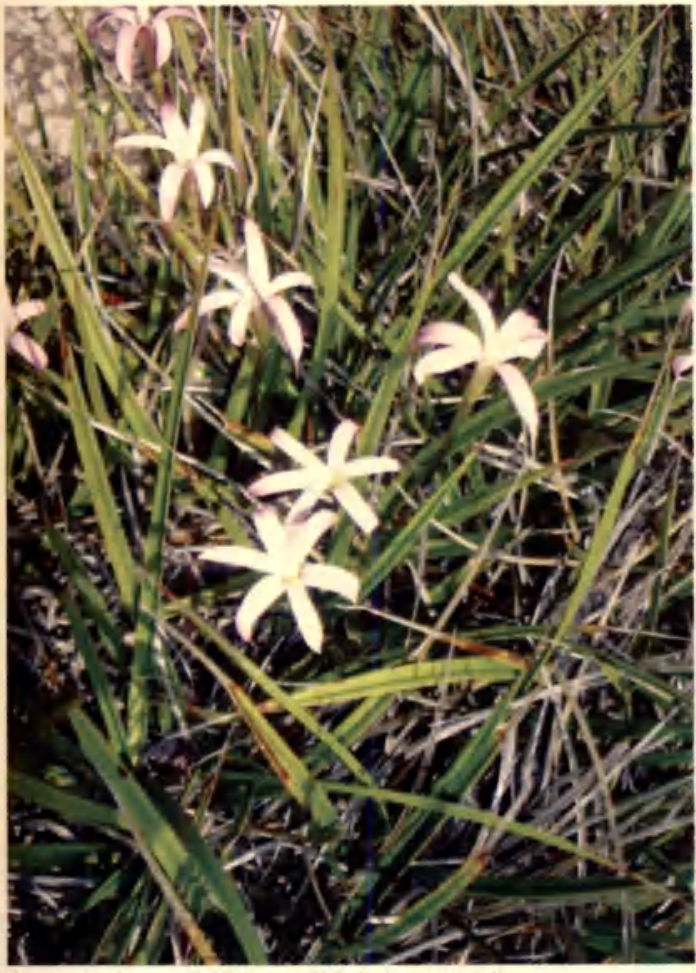

Vergasniopsis vargarii (Velloziat) "Machu hautag'ullay".

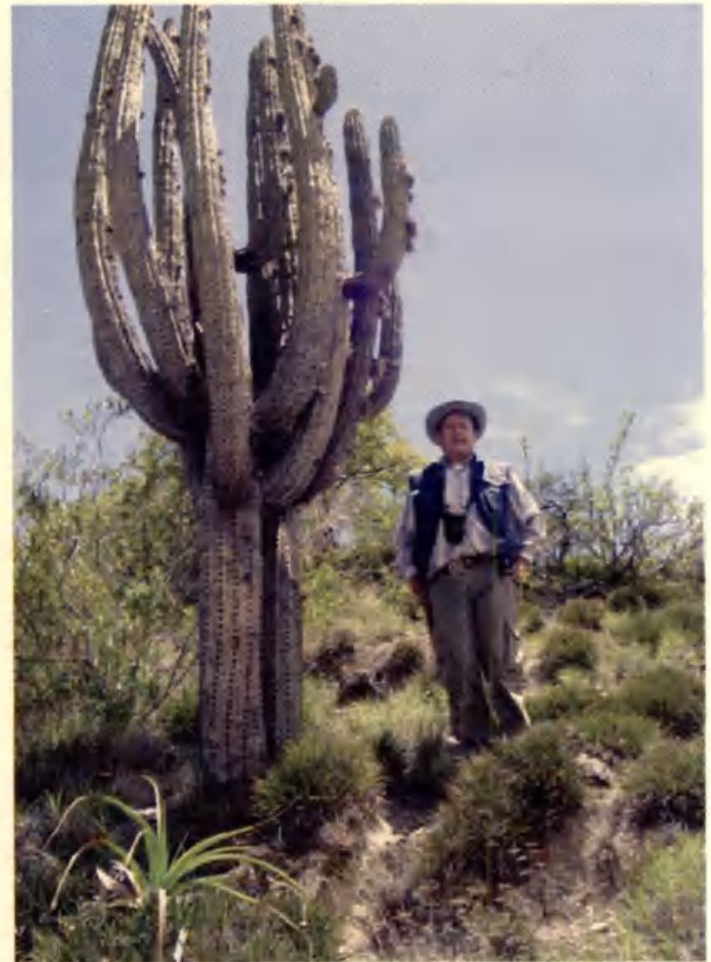

IVherhautorereus cuzcoensis (Cactactar).
Vish de Itamowata

Lamatamba an la magen izprierda de ro Bionco 
Revisfa UnJVersitaria 141

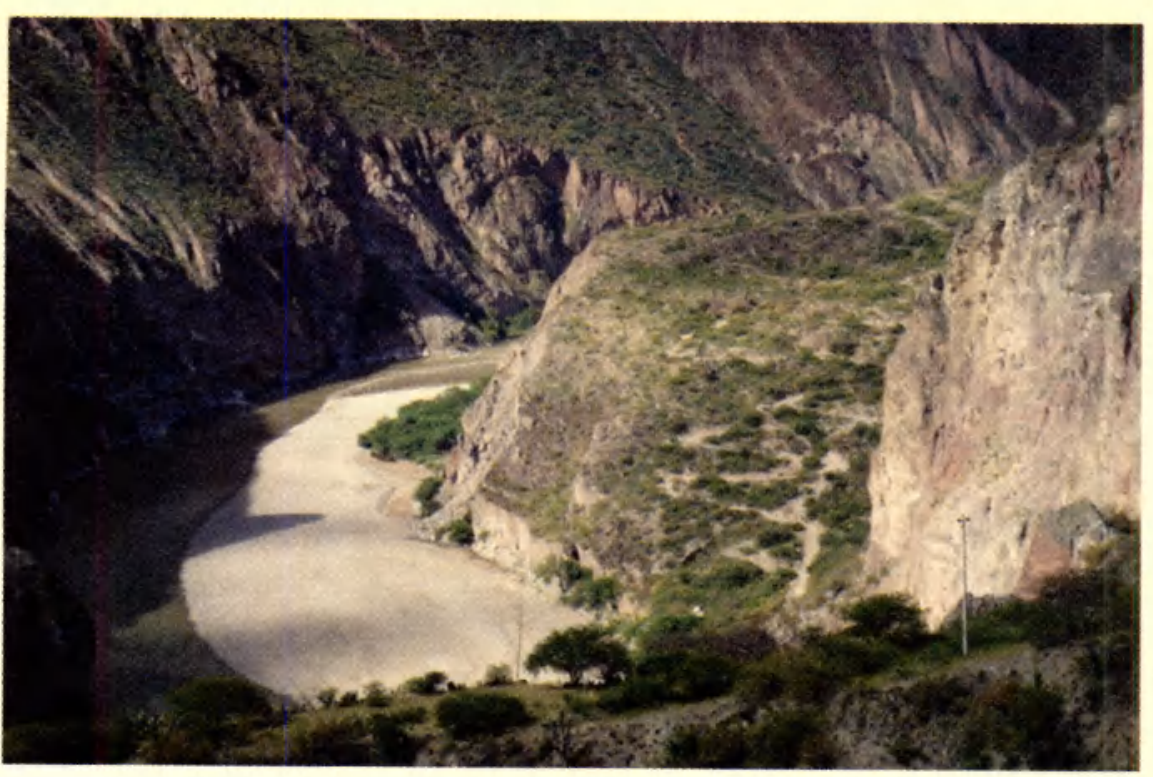

Vista de Qbnoq, bajamdo al no Apurimac

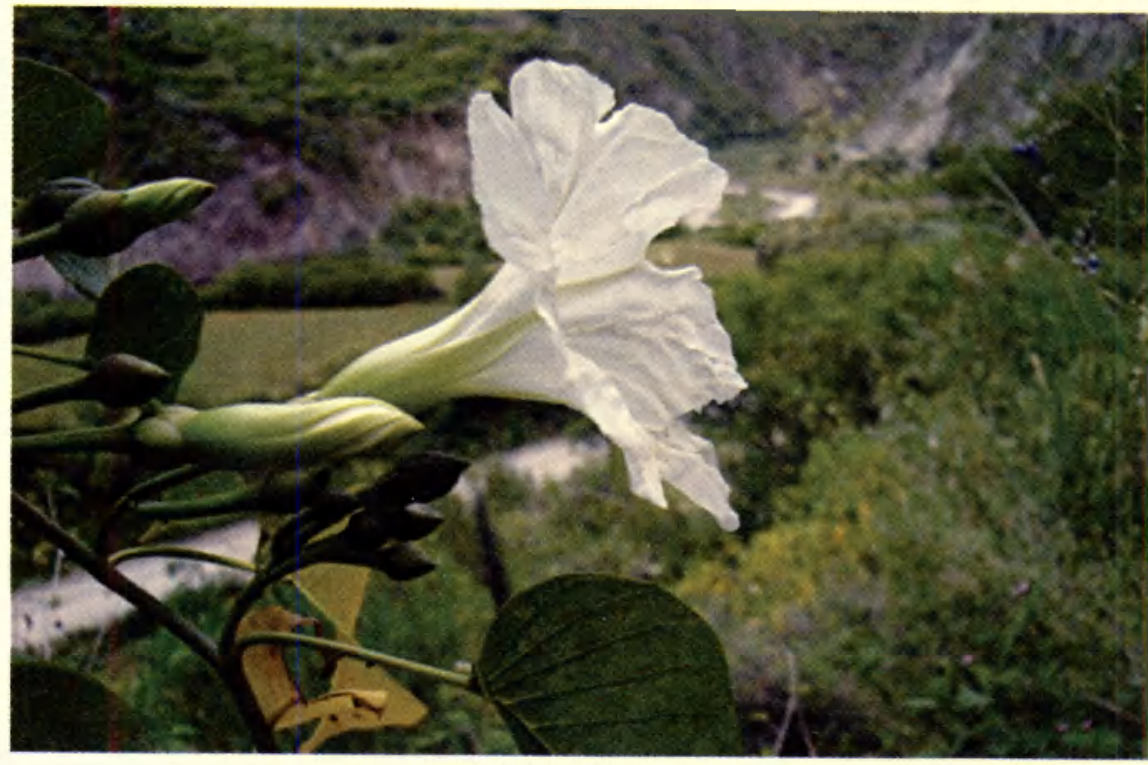

Ipomived carned

(Costrobtuthecee) "Wurag kamparillas".

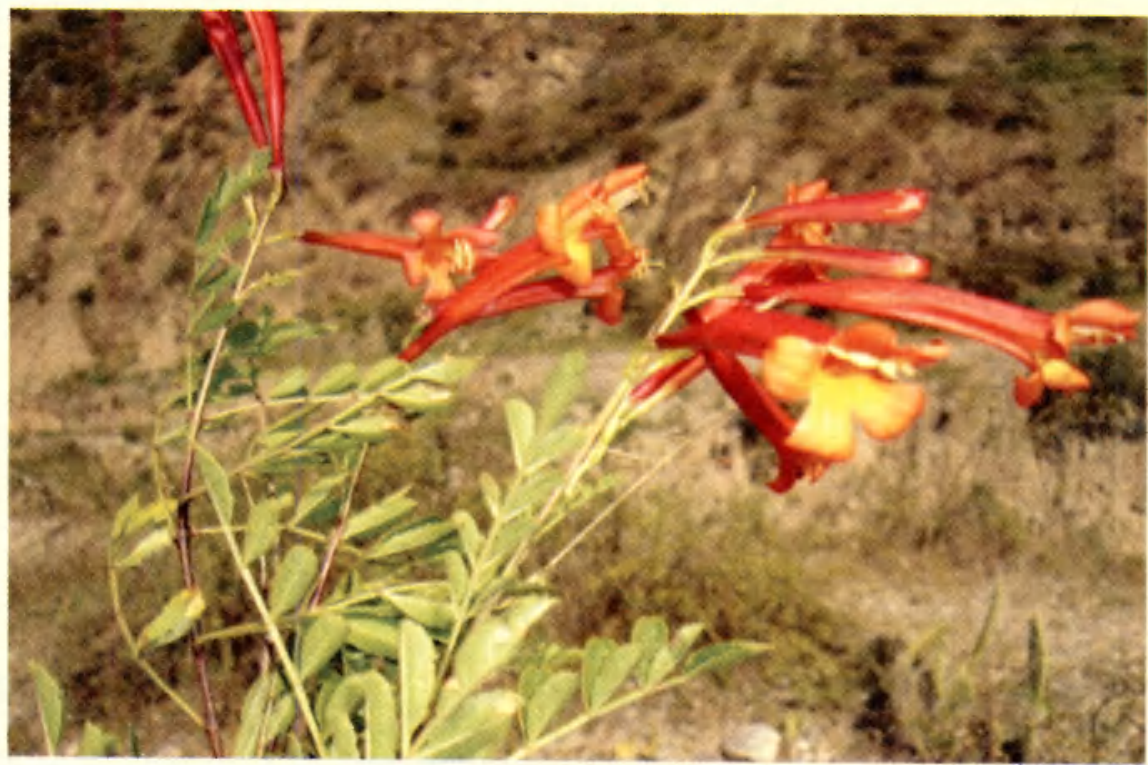



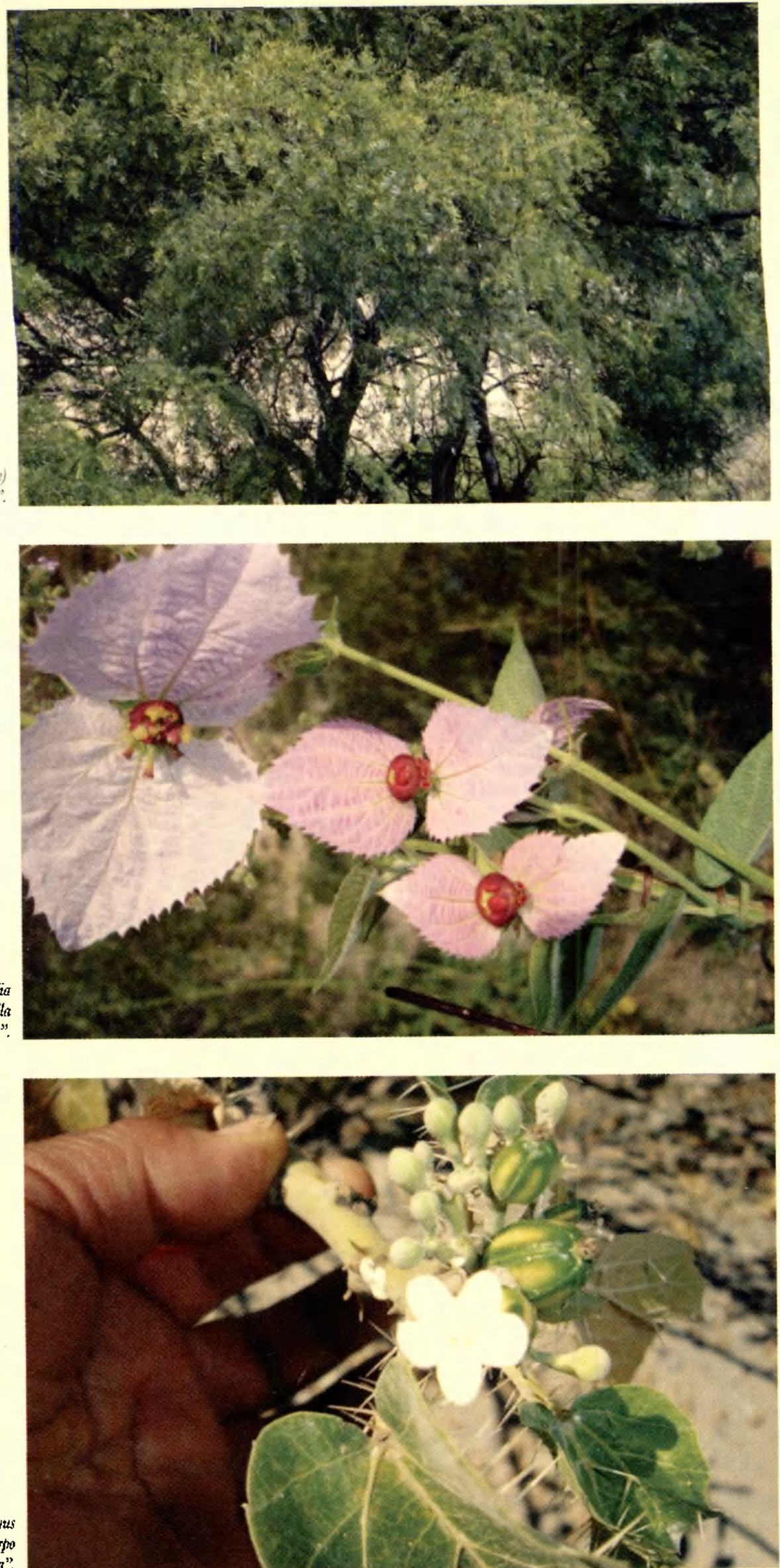


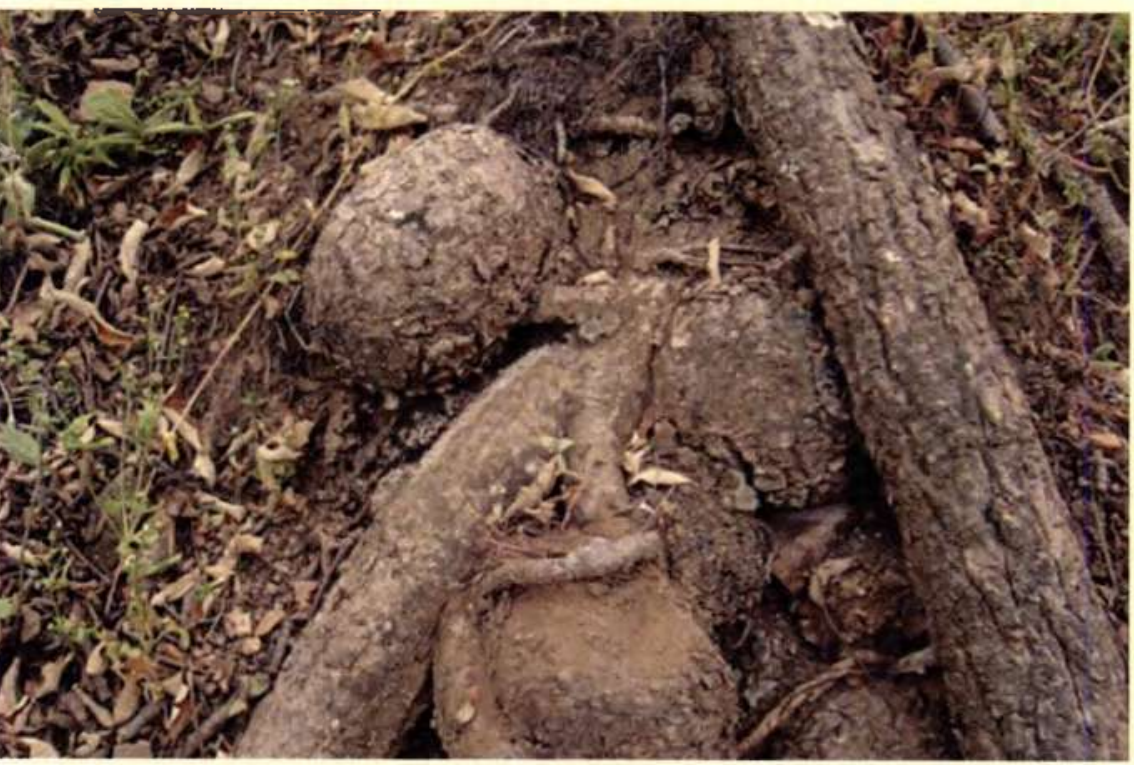

Formaciones xerotormas stublerraneas de Enotheca vargasii (Bombacacere) "P'ati-p'al".

\section{Bibliografía}

\section{ARESTEGUI, P. A.}

2004. Agroecosistemas en Curahuasi, Abancay, Apurimac. Cusco UNSAAC. Tesis para optar al Grado Académico de Magister en Ciencias

1996. "Distribución Altatudinal de los Fabaceas de Campac a Curntuass." En: Libro de resúmenes del VI CONABO'I, Cajamarca.

\section{BRACK A.}

1999. Dicrionario Encutlopedico de Plantas Útites del Peni. Cusco Centro do Estudios Regionales Andinos Bartolomé de las Casas.

2003. Pentí Diez mil añes de Domesticación. PMJJI Perú. FANPE gıtz. Lima, Edit. Bruño.

\section{BRAKO, L. \& J. ZARUCCHI}

1993. Calalogue of the Flowering Planis and Gymnosperms of Peni Monograf. Syst. Bot. Missouri Bot. Gard. 45.

\section{CHACóN, C: M. A.}

1988. Imponancia y Utildad de Kiziphts mistol (Rhannaceae) en th Alimentación Humana y Animal en Limalambo - Curnhtasi, Cusco, FEIU - UNSAAC.
1990. Utilidad de Prosopis aeberbateri en las Localdades de Limatamb - Curahasi. FEDU UNSAAC, Cusco.

\section{MATTEUCCI, S. D., COLMA, A.}

1982. Melodologia para el Estudio de la Vegelación. Secretaría General de la Organización dé los Estados Americanos, Washington.

\section{PEREZ, M.P.}

1989. Vegelación Fanerophyla del Valle de Limalambo. Cusco, Facultad de Ciencias Biológicas, UNSAAC. Tesis para optar al Título Profesional de Biólogo.

\section{SÁNCHEZ, R. 1.}

1985. Prayeto Erylhina edulis Triana, UNSAAC, Cusco, Convenio Perú Holanda, NUFFIC.

\section{WEBERBAUER, $\mathbf{A}$.}

1945. El Mundo Vegelal de los Andes Penuanos, Lima. www.fmnh.org

www.mobot.org 